\title{
CE-OFDM Schemes: Spectral Characterization and Optimum Performance
}

\author{
João Guerreiro ${ }^{1,3} \mathbb{D} \cdot$ Rui Dinis $^{1} \cdot$ Paulo Montezuma $^{2}$
}

Published online: 17 May 2017

(C) Springer Science+Business Media New York 2017

\begin{abstract}
Constant envelope orthogonal frequency division multiplexing (CE-OFDM) techniques have been recently proposed to allow the use of power-efficient saturated amplifiers, since those signals do not have the high peak-to-average ratio of OFDM signals, but maintain most of the OFDM advantages. However, the nonlinear nature of the phase modulation process inherent to CE-OFDM schemes gives rise to both theoretical challenges (analytical difficulties due to the nonlinear processes involved) and practical challenges (spectral widening and possible performance degradation due to nonlinear distortion effects). In this work, we present accurate expressions for the average power spectral density of CE-OFDM signals. Making use of recent results that indicate performance gains associated to the optimum detection of nonlinearly distorted multicarrier signals, we also investigate the optimum detection of CE-OFDM signals considering both additive white Gaussian noise channels and frequency-selective channels with Rayleigh fading, providing theoretical expressions for the asymptotic gains as well as the correspondent approximate bit-error-rate results.
\end{abstract}

Keywords Multicarrier signals · Nonlinear distortion · Optimum detection · Performance evaluation

João Guerreiro

jguerreiro.network@gmail.com

Rui Dinis

rdinis@fct.unl.pt

Paulo Montezuma

pmc@uninova.pt

Instituto de Telecomunicações, Lisboa, Portugal

2 UNINOVA, FCT-UNL, Caparica, Portugal

3 Universidade Autónoma de Lisboa, Lisboa, Portugal 


\section{Introduction}

Orthogonal frequency division multiplexing (OFDM) techniques [1] are very popular in the modern wireless digital communication standards. They are employed in several standards such as the long term evolution (LTE) [2], WiFi [3] and digital video broadcasting (DVB) [4]. This choice is mainly justified by their facility to combat the timedispersive nature of wireless channels even using low complex equalizers and their good spectral efficiency due to the orthogonal arrangement of the subcarriers. However, due to the very large envelope fluctuations of OFDM signals, they suffer from a high peak-toaverage power ratio (PAPR), which turns the amplification process a challenging task. Often, a large input back-off (IBO) is adopted to avoid nonlinear distortion in the transmitted signals. However, a large IBO compromises the power efficiency of the amplification process, which is a severe drawback especially for mobile terminals. To reduce the required IBO that assures a linear amplification, different PAPR-reducing techniques have been proposed. These PAPR-reducing techniques include clipping techniques [5, 6], multiple symbol representation techniques [7], partial transmit sequences (PTS) techniques [8] and many others (see [9, 10] and the references therein).

Although substantial PAPR reductions can be obtained, the resulting signals still have considerable envelope fluctuations and require the use of quasi-linear power amplifiers, although with smaller back-off. In fact, to avoid nonlinear distortion effects, the power amplifiers should be linear, at least in the region of the envelope's dynamic range. This precludes the use of very low cost and highly efficient power amplifiers that are strongly nonlinear such as class D, E or F amplifiers [11], since they require constant or quasiconstant envelope signals (i.e., signals with a PAPR close to $0 \mathrm{~dB}$ ). One of the first techniques that were proposed to address this challenge were the constant envelope paired burst OFDM (CEPB-OFDM) techniques [12], but they require very high oversampling factors and present a very poor spectral efficiency, which is not acceptable in wireless systems. Constant envelope (CE-OFDM) was recently proposed for mmWave communications [13-17]. As the name suggests, CE-OFDM signals have constant envelope, since they are generated by submitting a real-valued OFDM signal to a phase modulator. However, since the phase modulator is a nonlinear device, it can lead to substantial spectral widening due to the out of band radiation levels associated to intermodulation products (IMPs); it can also lead to significant bit-error-rate (BER) degradation due to in-band nonlinear distortion. Although receivers based on a phase modulator [14] achieve the optimum performance for low modulation indexes, this is not true for large modulation indexes, where the performance of these receivers is very poor and deviate considerably from the optimum performance $[15,16]$. In fact, recent results indicate that the nonlinear distortion component can have a positive effect on the BER performance, since it has information inherent to the transmitted symbols. In fact, by employing optimum or sub-optimum receivers the BER performance of OFDM schemes with strong nonlinear distortion effects can be as good or even better than the BER performance of linear OFDM schemes [18-20].

In this paper we take advantage of the Gaussian-like nature of OFDM signals for obtaining the analytical characterization of CE-OFDM signals and we present accurate power spectral density (PSD) estimates. Additionally, we study the asymptotic optimum performance associated to CE-OFDM signals in both ideal additive white Gaussian noise (AWGN) channels and frequency-selective channels with uncorrelated Rayleigh fading.

The paper has the following sections: CE-OFDM signals are discussed and characterized in Sect. 2. Section 3 concerns about the spectral characterization of CE-OFDM 
signals. Section 4 presents a study on the optimum performance of CE-OFDM schemes for both ideal AWGN channels, in Sect. 4.1, and for frequency-selective channels, in Sect. 4.2. Section 5 concludes this paper.

Throughout the paper we adopt the following notation: bold letters denote matrices or vectors and italic letters denote scalars. Capital letters are associated to the frequencydomain and small letters are associated to the time-domain. $\|\mathbf{X}\|$ denotes the Euclidean norm of the vector $\mathbf{X}$ and $(\cdot)^{T}$ denotes the transpose operator. The probability density function (PDF) of the random variable $x$ is denoted as $p(x)$ and $\mathbb{E}[\cdot]$ is the expectation operator.

\section{CE-OFDM System}

Figure 1 shows the CE-OFDM scheme adopted in this paper.

The vector $\mathbf{S}=\left[\begin{array}{lll}S_{0} S_{1} & \ldots S_{N-1}\end{array}\right]^{T} \in \mathbb{C}^{N}$ represents $N$ complex data symbols selected from a quadrature phase shift keying (QPSK) constellation with $S_{k}= \pm 1 \pm j$. We constrain the vector $\mathbf{S}=\left[\begin{array}{lll}S_{0} S_{1} \ldots S_{N-1}\end{array}\right]^{T} \in \mathbb{C}^{N}$ to have Hermitian symmetry as the phase modulator's input must be real-valued. In these conditions, the data symbols respect the following relation

$$
S_{k}=\left\{\begin{array}{cc}
0, & k=0, N / 2 \\
S_{N-k}=S_{k}^{*}, & \text { otherwise }
\end{array}\right.
$$

This means that only $N_{u}=(N-2) / 2$ are "useful" subcarriers. The block $\mathbf{S}=$ $\left[S_{0} S_{1} \ldots S_{N-1}\right]^{T} \in \mathbb{C}^{N}$ is further extended with $M(N-1)$ idle subcarriers at its edges, so as an oversampling operation by a factor of $M$ is obtained. This oversampling factor is employed not only to reduce the aliasing effects but also to obtain an adequate representation of the underlying continuous-time OFDM signal [21]. After an inverse discrete Fourier transform (IDFT), the correspondent time-domain samples are represented by $\mathbf{s}=\operatorname{IDFT}(\mathbf{S})=\left[\begin{array}{lll}\mathrm{s}_{0} \mathrm{~s}_{1} & \ldots & \mathrm{s}_{\mathrm{NM}-1}\end{array}\right]^{\mathrm{T}} \in \mathbb{C}^{\mathrm{NM}}$. We also employ a cyclic prefix $(\mathrm{CP})$ larger than the overall channel impulse response. Therefore, regarding the time-domain the effect of the frequency-selective channel is modeled by a single multiplication.

It is widely known that when the number of subcarriers is high, the time-domain samples of an OFDM signal $\mathbf{s}=\left[\begin{array}{lll}s_{0} s_{1} & \ldots & s_{N M-1}\end{array}\right]^{T} \in \mathbb{R}^{N M}$ have Gaussian nature and can be modelled by $s \sim \mathcal{N}\left(0, \sigma^{2}\right)$, with

$$
p(s)=\frac{1}{\sqrt{2 \pi \sigma^{2}}} \exp \left(-\frac{s^{2}}{2 \sigma^{2}}\right),
$$

where $\sigma^{2}=(N-2) /(N M)^{2}$. These time-domain samples are submitted to a phase modulator, whose output $\mathbf{y}=\left[\begin{array}{lll}y_{0} y_{1} & \ldots & y_{N M-1}\end{array}\right]^{T} \in \mathbb{C}^{N M}$ is characterized by

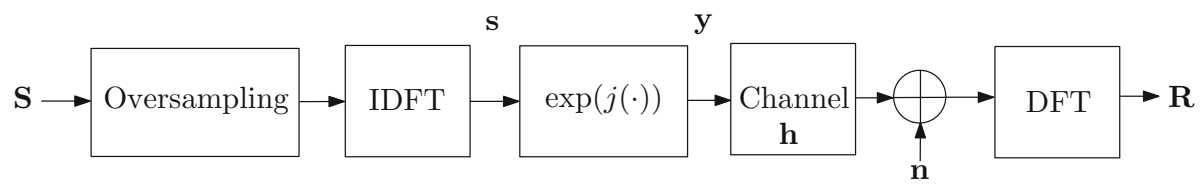

Fig. 1 CE-OFDM transmission scheme 


$$
y_{n}=f\left(s_{n}\right)=\exp \left(j 2 \pi h_{\sigma} s_{n}\right)=\sum_{l=0}^{+\infty} \frac{\left(j 2 \pi h_{\sigma} s_{n}\right)^{l}}{l !},
$$

where $h_{\sigma}=h / \sigma$ is the normalized modulation index.

Figure 2 shows the absolute value of the nonlinear function associated to the phase modulation process as well as its first order Taylor approximation [i.e., considering $l=1$ in (3)] for different modulation indexes. From the figure, it can be noted that when $2 \pi h$ is low, the first order Taylor approximation constitutes a good approximation of the phase modulation process. It is important to note that the term associated to $l=0$ consists in a DC component that does not carry any information. The degradation caused by this component is noticeable for low modulation indexes, where $\exp \left(j 2 \pi h_{\sigma} s_{n}\right) \approx 1+j 2 \pi h_{\sigma} s_{n}$ and cannot be reversed. Due to the Gaussian nature of OFDM signals, the Bussgang's theorem [22] applies. From this theorem, the output of a nonlinearity driven by a Gaussian signal can be written as the sum of two uncorrelated terms [23]: one that is proportional to the input and another associated to the nonlinear distortion. Therefore, the output of the phase modulator can be described as

$$
y_{n}=f\left(s_{n}\right)=\alpha s_{n}+d_{n},
$$

where the scale factor $\alpha$ is

$$
\alpha=\frac{\mathbb{E}\left[s_{n} f^{*}\left(s_{n}\right)\right]}{\mathbb{E}\left[s_{n}^{2}\right]}=\frac{\mathbb{E}\left[s_{n} f^{*}\left(s_{n}\right)\right]}{\sigma^{2}} .
$$

In the frequency-domain, the phase modulator output is given by $\mathbf{Y}=\operatorname{DFT}(\mathbf{y})=\left[\begin{array}{llll}\mathrm{Y}_{0} & \mathrm{Y}_{1} & \ldots & \mathrm{Y}_{\mathrm{NM}-1}\end{array}\right]^{\mathrm{T}}$, whose $k$ th element is

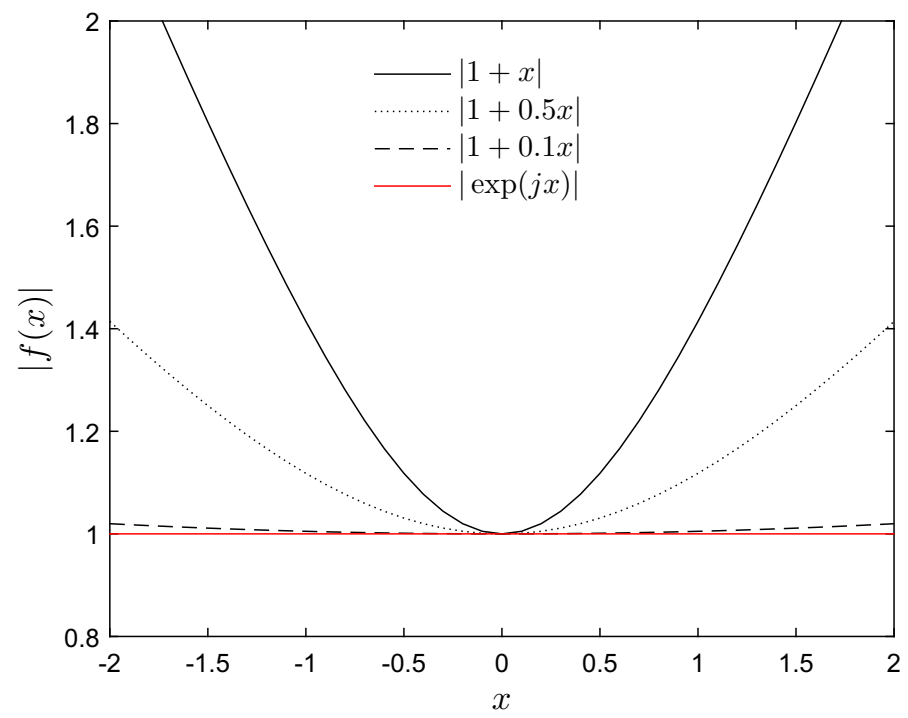

Fig. 2 Absolute value of $f(x)$ and its first order approximation considering different modulation indexes 


$$
Y_{k}=\alpha S_{k}+D_{k}
$$

where $D_{k}$ represents the distortion term associated to the $k$ th subcarrier. In our model, the CE-OFDM signal is submitted to a channel that can be either AWGN or frequencyselective. In both cases, the noise is characterized by the block $v=\operatorname{DFT}(\mathbf{n})=\left[\begin{array}{llll}v_{0} & v_{1} & \ldots & v_{\mathrm{NM}-1}\end{array}\right]^{\mathrm{T}} \in \mathbb{C}^{\mathrm{NM}}$, with $\mathbb{E}\left[\left|v_{k}\right|^{2}\right]=2 \sigma_{N}^{2}$. When the channel presents frequency selectivity, is can be modeled by the following impulse response

$$
h(t)=\sum_{i=0}^{I-1} \alpha_{i} \delta\left(t-\tau_{i}\right),
$$

where $I$ denotes the number of multipath components, $\alpha_{i}$ and $\tau_{i}$ are the power and the delay of the $i$ th ray, respectively, and $\delta(\cdot)$ is the Dirac delta function. The corresponding channel frequency response is the Fourier transform of $h(t)$, given by

$$
H(f)=\sum_{i=0}^{I-1} \alpha_{i} \exp \left(-j 2 \pi f \tau_{i}\right)
$$

The channel frequency response associated to the $k$ th subcarrier is $H_{k}=H(k / T)$, where $T$ represents the OFDM symbol duration. We model each one of the $I$ multipath components as a random variable with complex Gaussian distribution, i.e., $\alpha_{i} \sim \mathcal{C N}\left(0, \sigma_{i}^{2}\right)$. Moreover, to assure $H_{k} \sim \mathcal{C N}(0,1)$ and $\mathbb{E}\left[\left|H_{k}\right|^{2}\right]=1$, we define $\sigma_{i}^{2}=\frac{1}{I}$ for $i=0,1, \ldots, I-1$. Thanks to the $\mathrm{CP}$, the received signal in frequency-domain is represented by $\mathbf{R}=\left[\begin{array}{lll}R_{0} R_{1} & \ldots R_{N M-1}\end{array}\right]^{T} \in \mathbb{C}^{N M}$, whose $k$ th element is

$$
R_{k}=Y_{k} H_{k}+v_{k}=\alpha S_{k} H_{k}+D_{k} H_{k}+v_{k} .
$$

\section{Spectral Characterization of CE-OFDM Signals}

Here, we present an expression for the average PSD of CE-OFDM signals by taking the phase modulation process as a nonlinear transformation of a Gaussian signal. Following the approaches of [24-26], we firstly present the autocorrelation of the nonlinearly distorted OFDM signals at the phase modulator's output and then we obtain the average PSD using a Fourier transform.

Let us consider OFDM signals represented by a stationary, Gaussian random process $s(t)$, whose average PSD is $G_{s}(f)$. Due to the stationarity, the autocorrelation of the phase modulator's input, $R_{s}(\tau)$, only depends on the time lag between the two observation moments, $\tau$. Considering $s_{1}=s\left(t_{1}\right)$ and $s_{2}=s\left(t_{1}-\tau\right)$, we have $R_{s}(\tau)=\mathbb{E}\left[s_{1} s_{2}\right]$, while the power of the signal is $R_{s}(0)=\sigma^{2}$. At the phase modulator's output, the autocorrelation is

$$
R_{y}(\tau)=\mathbb{E}\left[f\left(s_{1}\right) f^{*}\left(s_{2}\right)\right]=\int_{-\infty}^{+\infty} \int_{-\infty}^{+\infty} f\left(s_{1}\right) f^{*}\left(s_{2}\right) p\left(s_{1}, s_{2}\right) \mathrm{ds}_{1} \mathrm{ds}_{2} .
$$

where $p\left(s_{1}, s_{2}\right)$ represents the joint PDF of the Gaussian random variables $s_{1}$ and $s_{2}$, and is given by 


$$
p\left(s_{1}, s_{2}\right)=\frac{1}{2 \pi \sigma^{2} \sqrt{1-\rho^{2}}} \exp \left(-\frac{s_{1}^{2}+s_{2}^{2}-2 \rho s_{1} s_{2}}{2 \sigma^{2}(1-\rho)}\right),
$$

with $\rho=\rho(\tau)=\frac{R_{s}(\tau)}{R_{s}(0)}$ denoting the normalized autocorrelation of the input signal. Considering the Mehler's formula [27], the joint PDF $p\left(s_{1}, s_{2}\right)$ can be rewritten using the marginal densities of $s_{1}$ and $s_{2}$ and the Hermite polynomials as

$$
p\left(s_{1}, s_{2}\right)=p\left(s_{1}\right) p\left(s_{2}\right) \sum_{m=0}^{+\infty} \frac{\rho^{m}}{2^{m} m !} H_{m}\left(\frac{s_{1}}{\sqrt{2} \sigma}\right) H_{m}\left(\frac{s_{2}}{\sqrt{2} \sigma}\right),
$$

where

$$
H_{m}(x)=(-1)^{m} \exp \left(x^{2}\right) \frac{d m}{d x^{m}}\left(\exp \left(-x^{2}\right)\right) .
$$

Therefore, we can rewrite (10) as

$$
\begin{aligned}
R_{y}(\tau) & =\int_{-\infty}^{+\infty} \int_{-\infty}^{+\infty} f\left(s_{1}\right) f\left(s_{2}\right) p\left(s_{1}\right) p\left(s_{2}\right) \sum_{m=0}^{+\infty} \frac{\rho^{m}}{2^{m} m !} \\
& \times H_{m}\left(\frac{s_{1}}{\sqrt{2} \sigma}\right) H_{m}\left(\frac{s_{2}}{\sqrt{2} \sigma}\right) \mathrm{ds}_{1} \mathrm{ds}_{2} .
\end{aligned}
$$

Further, by knowing that $p\left(s_{1}\right)=p\left(s_{2}\right)=p(s), f\left(s_{1}\right)=f\left(s_{2}\right)=f(s)$ [see (2) and (3)], and $H_{m}\left(\frac{s_{1}}{\sqrt{2} \sigma}\right)=H_{m}\left(\frac{s_{2}}{\sqrt{2} \sigma}\right)=H_{m}\left(\frac{s}{\sqrt{2} \sigma}\right)$, we have

$$
R_{y}(\tau)=\sum_{m=0}^{+\infty} \frac{\rho^{m}}{2^{m} m !} \mid \int_{-\infty}^{+\infty} \exp (j s) p(s) H_{m}\left(\frac{s}{\sqrt{2} \sigma}\right) \mathrm{d} s
$$

By defining $P_{m}$ as the power associated to the IMP of order $m$ as

$$
P_{m}=\frac{\left|\int_{-\infty}^{+\infty} \exp (j s) p(s) H_{m}\left(\frac{s}{\sqrt{2} \sigma}\right) \mathrm{d} s\right|^{2}}{2^{m} m !},
$$

we can finally rewrite (15) as

$$
R_{y}(\tau)=\sum_{m=0}^{+\infty} \rho^{m} P_{m}=\sum_{m=0}^{+\infty}\left(\frac{R_{s}(\tau)}{R_{s}(0)}\right)^{m} P_{m}
$$

Considering the Bussgang's theorem, the following separation can be done

$$
R_{y}(\tau)=R_{d c}+|\alpha|^{2} R_{s}(\tau)+R_{d}(\tau),
$$

where $R_{d c}$ and $R_{d}(\tau)$ are the autocorrelation terms related to distortion. By applying the Fourier transform to (18), the average PSD is 


$$
G_{y}(f)=R_{d c} \delta(f)+|\alpha|^{2} G_{s}(f)+G_{d}(f),
$$

where $R_{d c} \delta(f)$ is the deterministic component of the distortion (associated to the DC component), $G_{s}(f)$ denotes the PSD associated to the useful part of the signal and $G_{d}(f)$ is the PSD of the distortion component. To obtain the theoretical approximate PSD, the summation in (17) is truncated to $n_{\gamma}$, i.e., only the contribution of the first $n_{\gamma}+1$ IMPs is taken into account. This implicitly constitutes a polynomial approximation for the nonlinearity and the value of $n_{\gamma}$ that yields good accuracy depends on the modulation index, i.e., on the magnitude of the nonlinear distortion effects introduced by the phase modulator.

In the following of this section, we present a set of results regarding the average PSD of CE-OFDM signals. Figure 3 shows the average PSD of CE-OFDM signals obtained theoretically and by simulation for different values of $n_{\gamma}$. The modulation index is $2 \pi h=0.5$ and we consider OFDM signals with $N=128$ subcarriers and an oversampling factor of $M=4$. From the figure it can be noted that the accuracy of the theoretical method for obtaining the average PSD of CE-OFDM signals depends on the choice of $n_{\gamma}$. When $2 \pi h=0.5$ and $n_{\gamma}=5$, the difference between the theoretical and the simulated PSDs is almost $0 \mathrm{~dB}$. However, for higher modulation indexes and/or higher oversampling factors, the value of $n_{\gamma}$ required for good accuracy is higher too. This effect is illustrated in Fig. 4, where it is shown the average PSD of CE-OFDM signals considering $2 \pi h=1.8, n_{\gamma}=5$ and $n_{\gamma}=8, N=128$ and different oversampling factors. From the figure one can note that with this modulation index and $M=4,9$ IMPs are required for obtaining identical simulated and theoretical PSDs. However, when $M=8$ a value of $n_{\gamma}=8$ is not enough, and the PSDs do not match, especially in the out-of-band region. The power of the DC component and the total power of the distortion caused by the phase modulator is shown in Fig. , considering different modulation indexes. As $2 \pi h$ increases, the power of the DC component decreases, but the total distortion power increases since there are more nonlinear distortion terms. As mentioned before, nothing can be done to overcome the

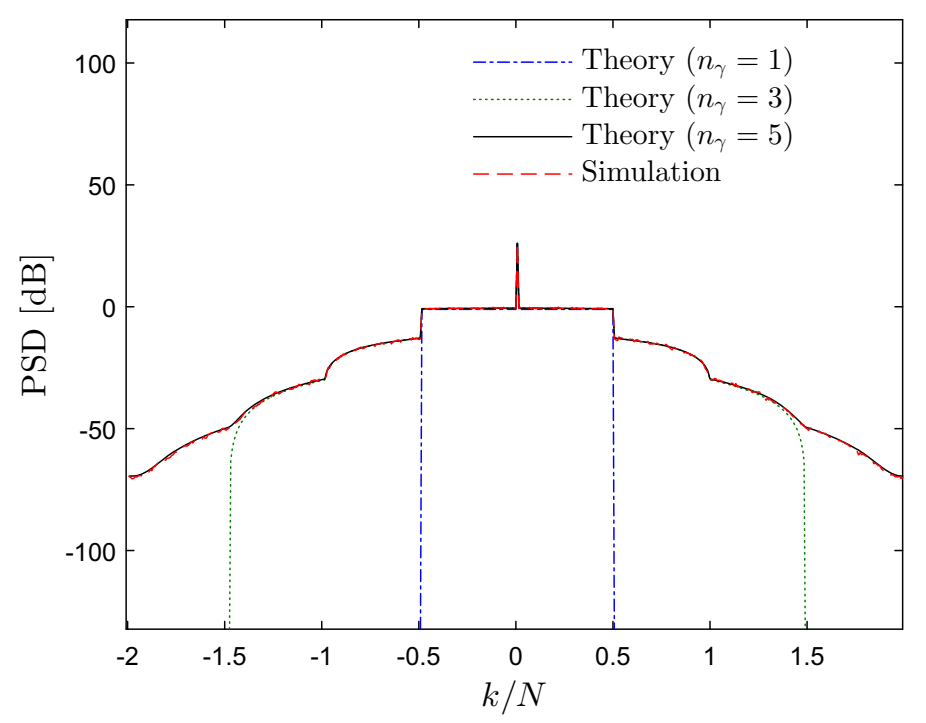

Fig. 3 Average PSD of a CE-OFDM signals obtained theoretically for different values of $n_{\gamma}$ and by simulation 

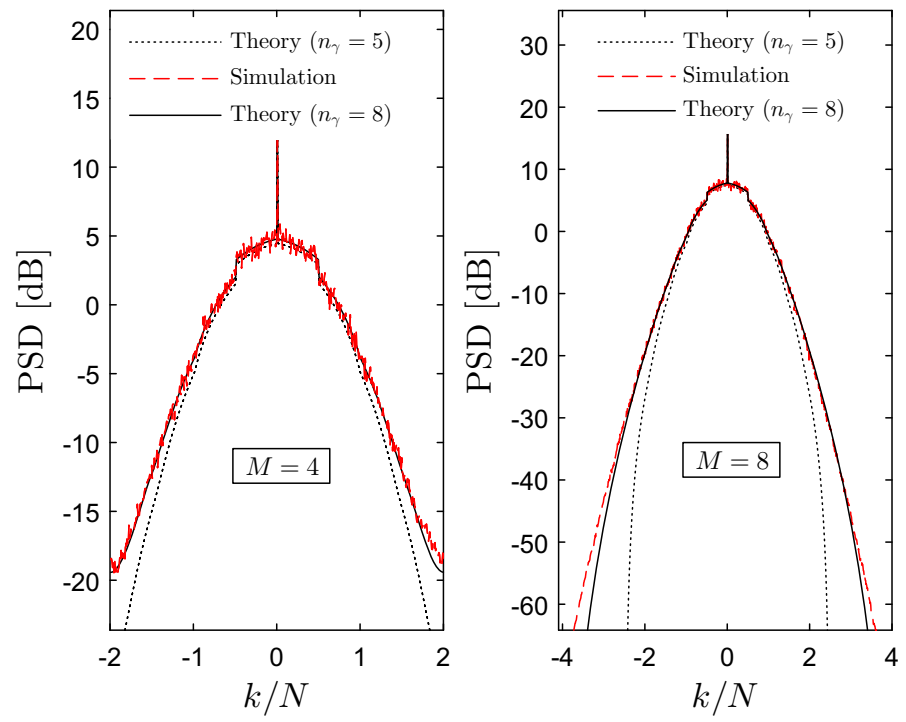

Fig. 4 Average PSD of CE-OFDM signals obtained for different values of $n_{\gamma}$ and by simulation considering $2 \pi h=1.8$ and $M=4$ (left figure) and $M=8$ (right figure)

degradation associated to the DC component. On the other hand, the degradation associated to the nonlinear distortion is noticeable when conventional OFDM receivers are taken into account, but less problematic for receivers based on a phase demodulator [14].

\section{Optimum Performance of CE-OFDM Signals}

It is widely known that multicarrier signals are often impaired by nonlinear distortion effects that can come either from a nonlinear amplification process or from nonlinear preprocessing techniques. Although a linear amplification can be made in CE-OFDM schemes due to the $0 \mathrm{~dB}$ PAPR of the signals, the OFDM signals are previously submitted to a phase modulator that introduces nonlinear distortion effects. The unwanted effects of this distortion are twofold: (1) it causes in-band distortion that leads to BER degradations, and (2) out-of-band radiation that can increase the adjacent channel interference (ACI) levels. While the latter can be mitigated through filtering operations, the former is conventionally seen as an additive noise term, which is a consequence of the Bussgang's theorem [23]. This motivated the development of Bussgang noise cancellation (BNC) receivers [28, 29], whose goal is estimate and cancel the in-band nonlinear, additive noise. However, the performance of these receivers is not good and they can even perform worse than the conventional receivers, especially when the signal to noise ratio (SNR) is low.

Recently, another point of view was given to the nonlinear distortion. In [18-20] it was demonstrated that the nonlinear distortion has information on the transmitted signals and, instead of being regarded as noise, it can be considered to be useful information that can be harvested by optimum receivers. The optimum detection can provide two types of gain: an energy gain and a diversity gain that can be explored in frequency-selective channels. The main disadvantage associated to this type of detection is its very high complexity that is associated with the large number of comparisons made by the receiver even when $N$ is 
small and/or QPSK constellations are employed. However, it is still important to verify if there are asymptotic gains associated to the optimum detection of CE-OFDM signals. In order to do that, we focus our attention in the computation of an approximation of the pairwise error probability (PEP), that is related to the squared Euclidean distance between two CE-OFDM signals and can provide an insight to the optimum performance. In this section, we study the squared Euclidean distance between two CE-OFDM signals in both AWGN and frequency-selective channels, and present the correspondent asymptotic BER making use of the results of $[19,20]$.

\subsection{AWGN Channels}

Our goal is to derive the squared Euclidean distance between two CE-OFDM signals $\mathbf{R}^{(2)}$ and $\mathbf{R}^{(1)}$ [see (9)]

$$
D_{n l}^{2}=\left\|\mathbf{R}^{(2)}-\mathbf{R}^{(1)}\right\|^{2}
$$

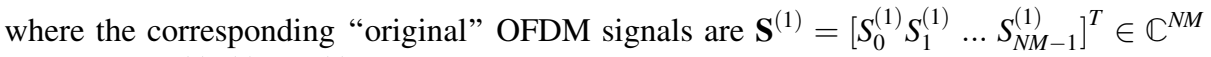
and $\mathbf{S}^{(2)}=\left[S_{0}^{(2)} S_{1}^{(2)} \ldots S_{N M-1}^{(2)}\right]^{T} \in \mathbb{C}^{N M}$. $\mathbf{S}^{(2)}$ and $\mathbf{S}^{(1)}$ signals differ in $\mu$ bits at the indexes represented by the set $\Phi=\left[\Phi_{0} \Phi_{1} \ldots \Phi_{\mu-1}\right]$, where $\Phi_{i} \in\{0, N-1\}$. It is important to mention that when these blocks differ in $\mu$ bits, they have $2 \mu$ different subcarriers, which is a consequence of the Hermitian symmetry. This effect is illustrated in Fig. 6 that shows the squared Euclidean distance between two CE-OFDM signals considering $\mu=1, N=512$, $M=4$ and a modulation index of $2 \pi h=1.0$.

The squared Euclidean distance between $\mathbf{S}^{(1)}$ and $\mathbf{S}^{(2)}$ is $D^{2}=\left\|\mathbf{S}^{(2)}-\mathbf{S}^{(1)}\right\|^{2}=8 \mu E_{b}$, where $E_{b}=\sum_{k=0}^{N M-1}\left|S_{k}\right|^{2} / N_{u}$ is the average bit energy. Considering (9), we can expand (20) as $^{1}$

$$
\begin{aligned}
D_{n l}^{2} & =\sum_{k=0}^{M N-1}\left|H_{k}\right|^{2}\left|\alpha\left(S_{k}^{(2)}-S_{k}^{(1)}\right)+D_{k}^{(2)}-D_{k}^{(1)}\right|^{2} \\
& =\sum_{k=0}^{M N-1}\left|\alpha\left(S_{k}^{(2)}-S_{k}^{(1)}\right)+D_{k}^{(2)}-D_{k}^{(1)}\right|^{2},
\end{aligned}
$$

since for AWGN channels, $\left|H_{k}\right|^{2}=1 \forall k$. The asymptotic gain associated to the optimum detection is given by

$$
G=\frac{D_{n l}^{2}}{4 E_{b}}
$$

Clearly, when the transmission is linear we have $D_{n l}^{2}=D^{2}=4 E_{b}$ and $G=1$. It was shown in [20] that average value of (22) considering real-valued, Gaussian multicarrier signals can be theoretically obtained as

\footnotetext{
${ }^{1}$ Here, we neglect the effect of $v_{k}$ since $\mathbb{E}\left[v_{k}\right]=0$.
} 


$$
\mathbb{E}[G]=\frac{\mathbb{E}\left[D_{n l}^{2}\right]}{4 E_{b}}=\frac{\mu d_{\mathrm{adj}}^{2} \int_{-\infty}^{+\infty}\left|f^{\prime}(s)\right|^{2} p(s) \mathrm{d} s}{2 \int_{-\infty}^{+\infty}|f(s)|^{2} p(s) \mathrm{d} s},
$$

where $d_{\text {adj }}^{2}$ is the squared absolute value of the difference between two adjacent QPSK symbols, $f(s)$ is the nonlinear function associated to the phase modulator that is represented in (3) and $f^{\prime}(s)$ is its derivative.

Figure 7 shows the average asymptotic gain associated to the optimum detection of CEOFDM signals obtained theoretically with (23) and by simulation averaging (22) over a large number of sequences and error positions. The OFDM signals have different values of $N, M=4$ and several modulation indexes $2 \pi h$ are considered.

From the figure, it can be noted that the accuracy of (23) increases with the number of subcarriers, however, even for relatively small $N$, the accuracy is relatively high. As expected, the modulation index plays a very important role in the magnitude of the average asymptotic gain associated to the optimum performance of CE-OFDM signals. Indeed, for $2 \pi h<1.0$, we actually have a degradation. However, when $2 \pi h>1.0$ there are considerable gains. It is worth to mention that when the modulation index $2 \pi h$ increases, the nonlinear distortion effects increase and the power correspondent to the DC component introduced by the phase modulator decreases (see Fig. 5), which explains the existence of higher gains. As mentioned before, the results of Fig. 7 were obtained considering only a portion of all the possible transmitted sequences. However, the true PEP associated to the optimum detection of CE-OFDM signals require the computation of the squared Euclidean distance between the received signal and the possible transmitted signals, which involves

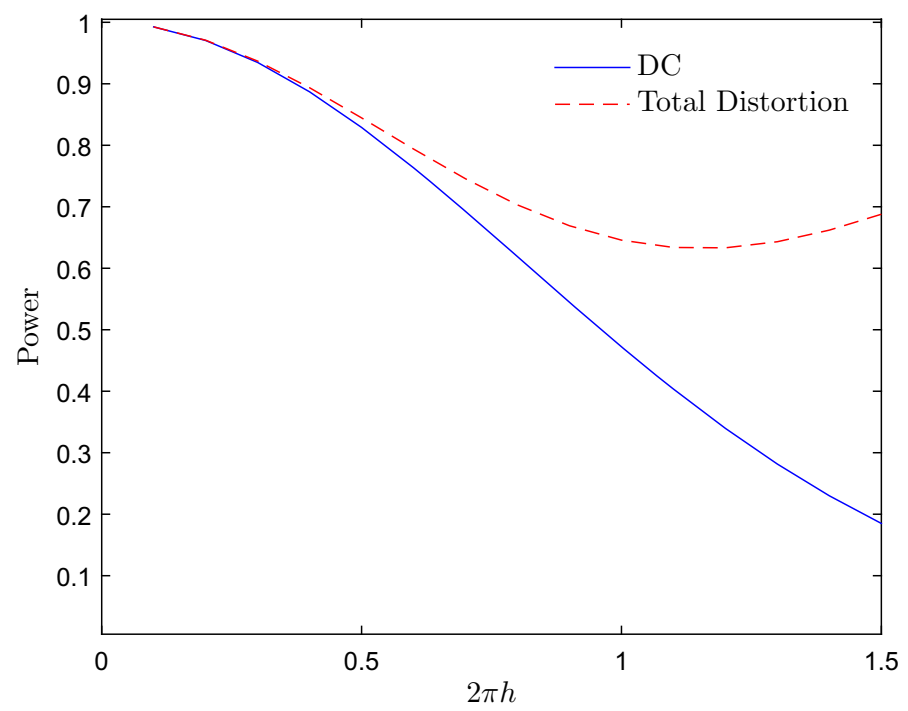

Fig. 5 Evolution of the power associated to the DC component and the total distortion power with the modulation index 
an extremely large complexity. For this reason, we obtain an approximation of the PEP that gives the asymptotic BER associated to the optimum detection of CE-OFDM signals. This approximate BER is computed by evaluating the squared Euclidean distance between a given sequence $\mathbf{S}^{(1)}=\left[S_{0}^{(1)} S_{1}^{(1)} \ldots S_{N M-1}^{(1)}\right]^{T} \in \mathbb{C}^{N M}$ and all its possible variations of $\mu=1$ bits represented by $\mathbf{S}^{(2)}=\left[S_{0}^{(2)} S_{1}^{(2)} \ldots S_{N M-1}^{(2)}\right]^{T} \in \mathbb{C}^{N M}$, i.e.,

$$
P_{b} \approx \mathbb{E}_{\mathbf{S}^{(1)}}\left[\frac{1}{2 N} \sum_{\mathbf{S}^{(2)} \neq \mathbf{S}^{(1)}} Q\left(\sqrt{\frac{\left\|\mathbf{Y}^{(2)}-\mathbf{Y}^{(1)}\right\|^{2}}{2 N_{0}}}\right)\right],
$$

with $N_{0}$ representing the noise PSD. When the transmission is linear, there is no energy gain associated to the optimum detection. In these conditions, it is easily observed that $\left\|\mathbf{Y}^{(2)}-\mathbf{Y}^{(1)}\right\|^{2}=\left\|\mathbf{S}^{(2)}-\mathbf{S}^{(1)}\right\|^{2}=4 E_{b}$. Consequently, the Eq. (24) reduces to BER expression for the linear transmission of QPSK constellations in AWGN channels, i.e., $P_{b}=Q\left(\sqrt{\frac{2 E_{b}}{N_{0}}}\right)$.

Figure 8 presents the optimum asymptotic BER obtained with (24) considering a large number of random OFDM signals with $N=256, M=4$ and different modulation indexes. From the figure, we note that when $2 \pi h=0.5$ and $P_{b}=10^{-3}$ there is a degradation of approximately $6 \mathrm{~dB}$, which was expected due to the very high power associated to the DC component for this modulation index. When $2 \pi h=1.0$, there is actually neither a gain nor a degradation, since the approximate PEP is equal to the BER curve for a linear transmission. As said before, the asymptotic gains appear for values of $2 \pi h>1.0$, which can be verified for the approximate BER curve when $2 \pi h=1.5$, where the gain at $P_{b}=10^{-3}$ is around $3.6 \mathrm{~dB}$ (see Fig. 9).

\subsection{Frequency-Selective Channels}

In this subsection we analyze the optimum performance of CE-OFDM schemes considering linear time-invariant channels that present frequency-selective Rayleigh fading. Again, our goal is to compute the squared Euclidean distance between $\mathbf{R}^{(1)}=$ $\left[R_{0}^{(1)} R_{1}^{(1)} \ldots R_{N M-1}^{(1)}\right]^{T} \in \mathbb{C}^{N M}$ and $\mathbf{R}^{(2)}=\left[R_{0}^{(2)} R_{1}^{(2)} \ldots R_{N M-1}^{(2)}\right]^{T} \in \mathbb{C}^{N M}$. However, unlike it was done in (21), we must take into account the channel frequency responses $\mathbf{H}=\left[\begin{array}{llll}H_{0} & H_{1} & \ldots & H_{M N-1}\end{array}\right]^{T} \in \mathbb{C}^{N M}$. We define the Euclidean distance in these conditions as

$$
D_{n l, h}^{2}=\sum_{k=0}^{M N-1}\left|H_{k}\right|^{2}\left|\alpha\left(S_{k}^{(2)}-S_{k}^{(1)}\right)+D_{k}^{(2)}-D_{k}^{(1)}\right|^{2},
$$

and the potential asymptotic gain as

$$
\begin{gathered}
G_{h}=\frac{D_{n l, h}^{2}}{4 E_{b, h}} \\
=\frac{\sum_{k=0}^{M N-1}\left|H_{k}\right|^{2}\left|\alpha\left(S_{k}^{(2)}-S_{k}^{(1)}\right)+D_{k}^{(2)}-D_{k}^{(1)}\right|^{2}}{4 E_{b}},
\end{gathered}
$$

where it should be noted that $\mathbb{E}_{h}\left[E_{b, h}\right]=E_{b}$. In fact, the value of this gain is now not only dependent on the data sequences but also on the statistical characterization of the channel 


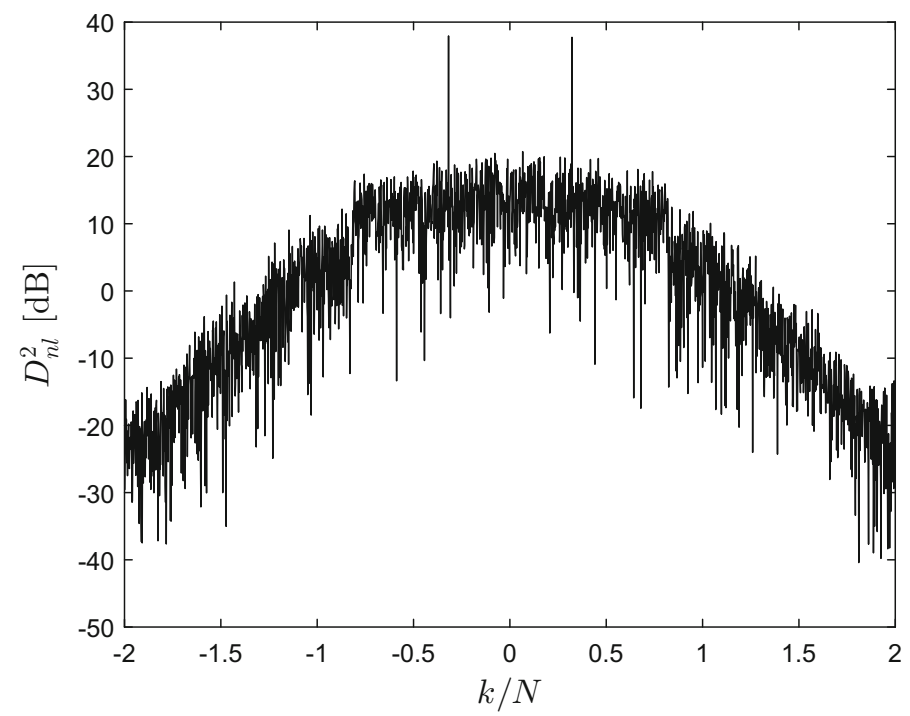

Fig. 6 Squared Euclidean distance between two CE-OFDM signals considering $2 \pi h=1.0$

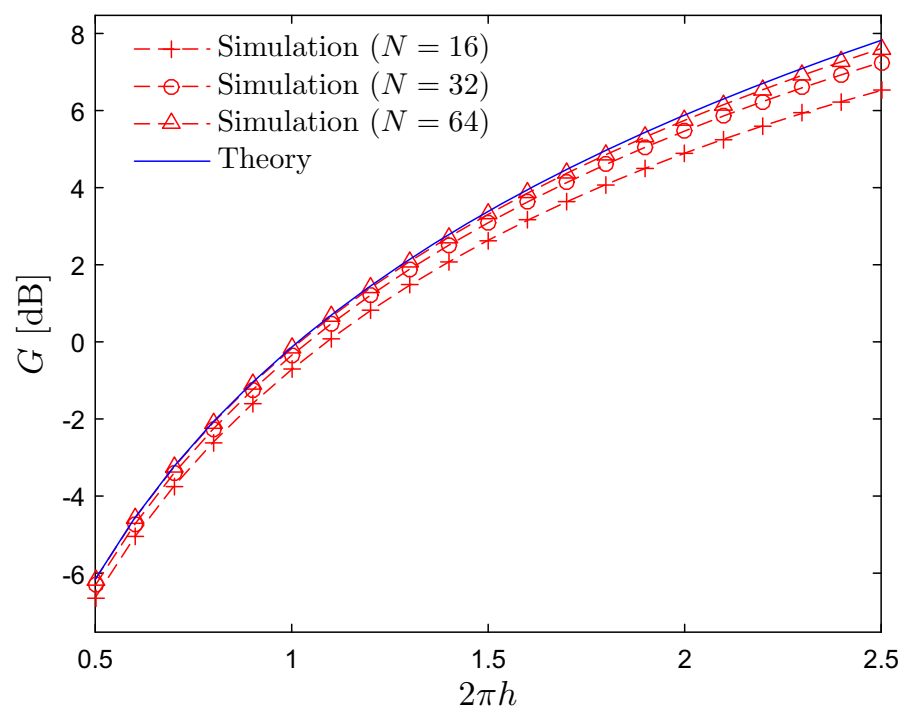

Fig. 7 Asymptotic gain associated to the optimum detection of CE-OFDM signals obtained both by simulation and theoretically for different values of $N$

frequency responses. Considering Fig. 6 it can be noted that the distance have two mainly components: one that is associated to the erroneous subcarriers and another that is associated to the difference between the nonlinear distortion terms. Using this fact, we can rewrite (25) 


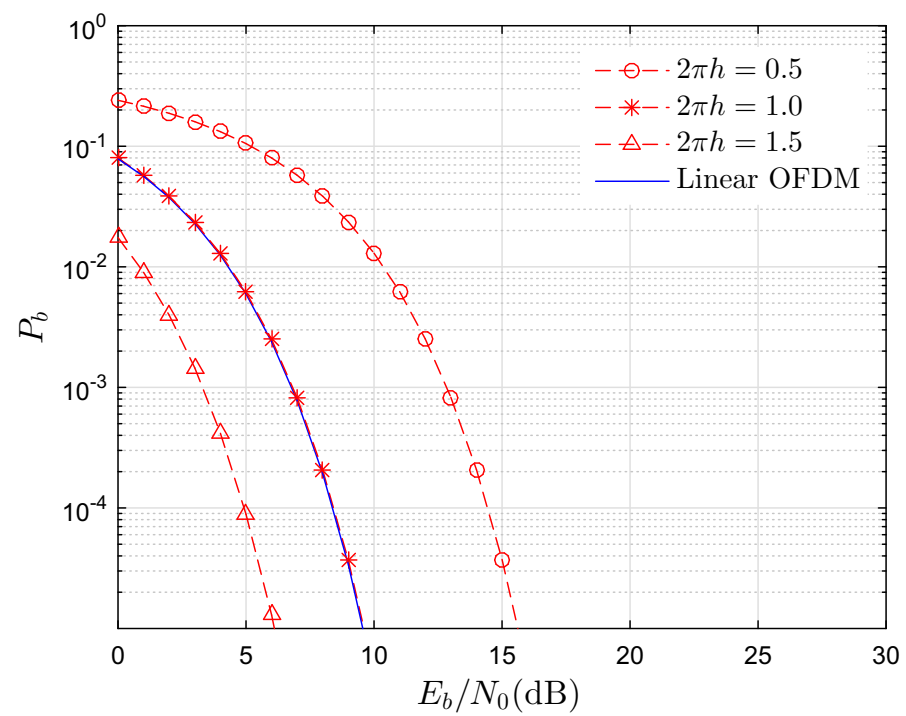

Fig. 8 Approximate BER associated to the optimum detection of CE-OFDM signals considering different modulation indexes

$$
\begin{aligned}
D_{n l, h}^{2}= & \sum_{k=0}^{M N-1}\left|H_{k}\right|^{2}\left|\alpha\left(S_{k}^{(2)}-S_{k}^{(1)}\right)+D_{k}^{(2)}-D_{k}^{(1)}\right|^{2} \\
\approx & \sum_{k=0}^{M N-1}\left|H_{k}\right|^{2}\left(\left|\alpha\left(S_{k}^{(2)}-S_{k}^{(1)}\right)\right|^{2}+\mathbb{E}\left[\left|D_{k}^{(2)}-D_{k}^{(1)}\right|^{2}\right]\right) \\
= & \underbrace{\sum_{k \in \Phi}\left|H_{k}\right|^{2}\left|\alpha\left(S_{k}^{(2)}-S_{k}^{(1)}\right)\right|^{2}}_{D_{n l, h_{d}}^{2}} \\
& +\underbrace{\sum_{k=0}^{M N-1}\left|H_{k}\right|^{2} \mathbb{E}\left[\left|D_{k}^{(2)}-D_{k}^{(1)}\right|^{2}\right]}_{D_{n l, h_{c}}^{2}}
\end{aligned}
$$

with $D_{n l, h_{c}}^{2}$ denoting the "continuous" part of the distance, and $D_{n l, h_{d}}^{2}$ denoting the "discrete" part of the distance. By considering this definition for the squared Euclidean distance, we also can redefine the asymptotic gain in (26) as the sum of two distinct gain components

$$
G_{h}=\frac{D_{n l, h_{c}}^{2}}{4 E_{b}}+\frac{D_{n l, h_{d}}^{2}}{4 E_{b}}=G_{h_{c}}+G_{h_{d}} .
$$

Due to the random nature of $\left|H_{k}\right|^{2}$, both $G_{h c}$ and $G_{h d}$ (and consequently $G_{h}$ ) are also random and they need to be analysed statistically. In [20], the distribution of $G_{h}$ for realvalued OFDM signals was derived and it was shown to be 


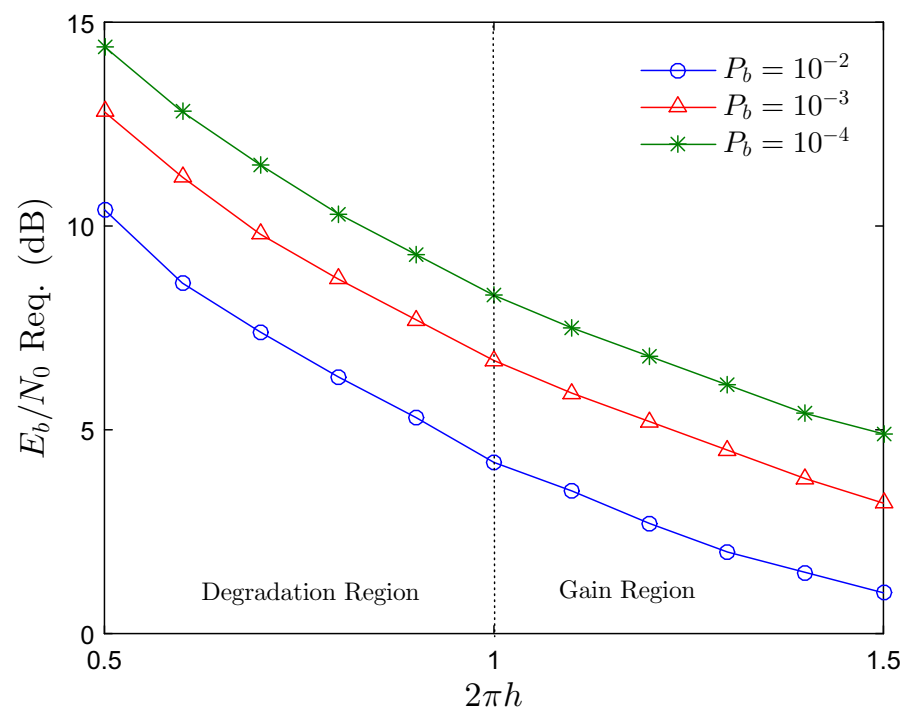

Fig. 9 Required values of $E_{b} / N_{0}$ to achieve different values of $P_{b}$ considering different values of $2 \pi h$

$$
\begin{aligned}
& p\left(G_{h}\right)=\frac{\left(\frac{\mu}{\mathbb{E}\left[G_{h d}\right]}\right)^{\mu}\left(\frac{I}{\mathbb{E}\left[G_{h c}\right]}\right)^{I}}{\Gamma(I+\mu)} G_{h}^{I+\mu-1} \exp \left(-\frac{G_{h} I}{\mathbb{E}\left[G_{h c}\right]}\right) \\
& \times M\left(\mu, I+\mu, G_{h}\left(\frac{I}{\mathbb{E}\left[G_{h c}\right]}-\frac{\mu}{\mathbb{E}\left[G_{h d}\right]}\right)\right),
\end{aligned}
$$

with $M(a, b, z)$ denoting the Kummer's function of the first kind [30].

Figure 10 shows $p\left(G_{h}\right)$ both by simulation and theoretically using (30). The CE-OFDM signals have $N=128, M=4$ and different values of $2 \pi h$ are considered. The frequencyselective channel has $I=32$ multipath rays. From the figure it can be noted that (30) presents good accuracy. As in the case of ideal AWGN channels, for higher modulation indexes we have higher potential asymptotic gains. When $2 \pi h=0.5$, it is very likely that $G_{h}$ is lower than 1 which is traduced in poor performance relatively to the conventional, linear OFDM schemes. This can be explained due to the power that is "wasted" in the DC component that does not have any information about the transmitted signals.

Figure 11 shows the approximate PEP for CE-OFDM signals in frequency-selective channels. It is obtained considering the distribution of the gain $p\left(G_{h}\right)$, OFDM signals with $N=256, M=4$ and several modulation indexes. As expected, there are considerable performance improvements. When $2 \pi h=1.0$, the performance is substantially better than the performance associated to the linear, OFDM schemes. The required $E_{b} / N_{0}$ for $P_{b}=$ $10^{-4}$ is $6 \mathrm{~dB}$ for $2 \pi h=1.5,10.5 \mathrm{~dB}$ for $2 \pi h=1.0$, and $13.8 \mathrm{~dB}$ for $2 \pi h=0.75$, which compares with approximately $20 \mathrm{~dB}$ when the transmission is linear. To promote a "fair" comparison, the linear OFDM BER curve considers diversity of order $L=2$, since due to the Hermitian symmetry of the CE-OFDM data block, an additional diversity effect is introduced. 


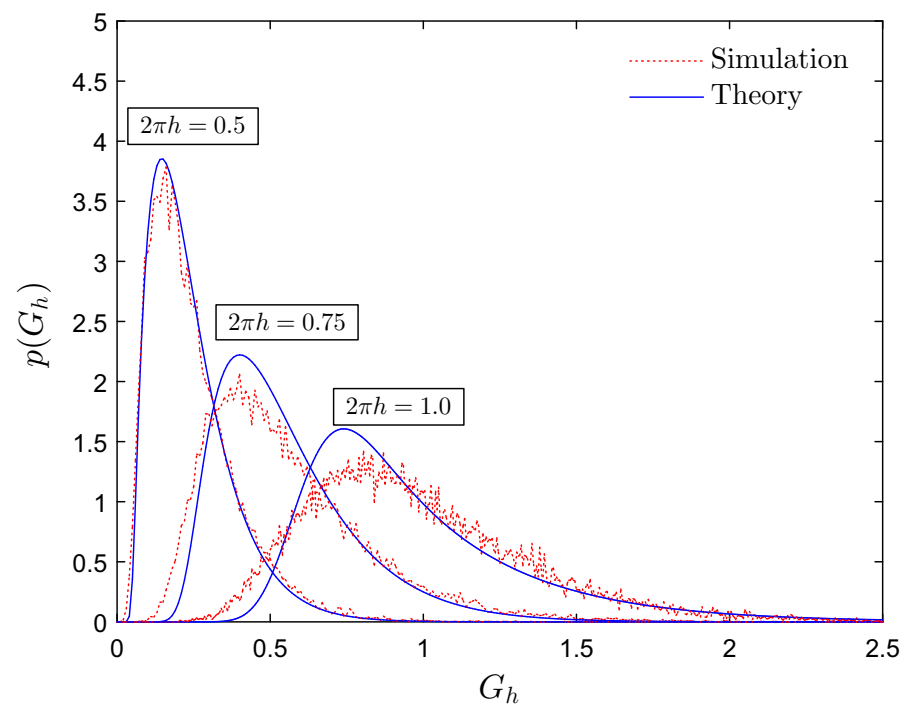

Fig. 10 Distribution of the asymptotic gain associated to the optimum detection of CE-OFDM signals in frequency-selective channels

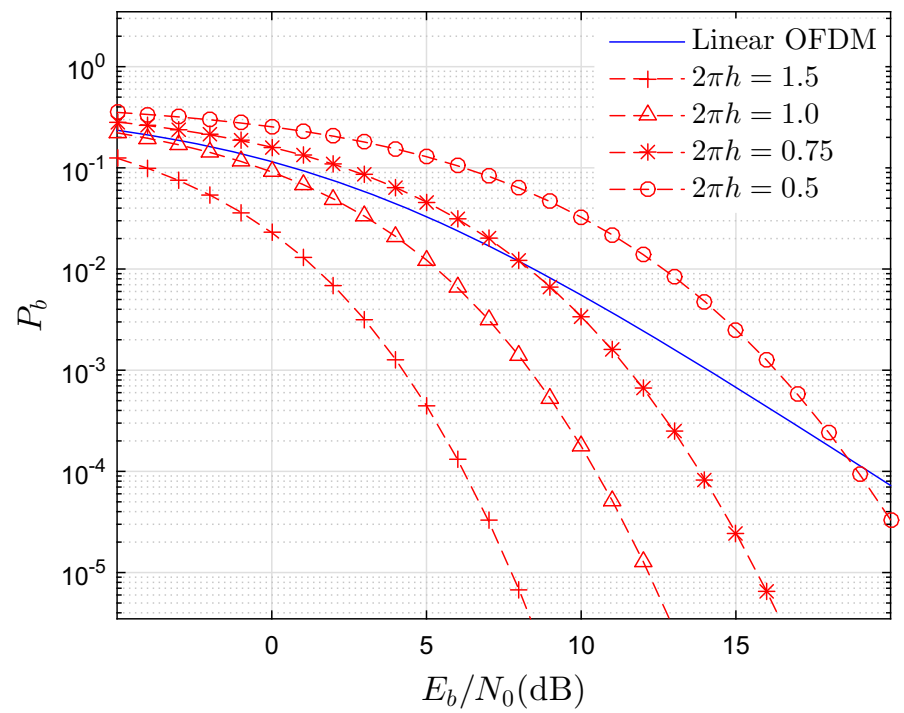

Fig. 11 Approximate BER associated to the optimum detection of CE-OFDM signals in frequencyselective channels considering different modulation indexes

\section{Conclusions}

In this work we present an accurate spectral characterization of CE-OFDM signals by making use of well established results on the nonlinear transformation of Gaussian signals. Moreover, we also study the optimum performance of CE-OFDM signals in both ideal 
AWGN and frequency selective channels, and we confirm the existence of large potential asymptotic gains by presenting accurate theoretical expressions for the asymptotic gain. It was shown that these asymptotic gains are noticeable for frequency-selective channels since in this type of channels the optimum detection can harvest the diversity effect introduced by the CE-OFDM modulation.

Acknowledgements This work was supported in part by Fundação para a Ciência e Tecnologia (FCT) and Instituto de Telecomunicações under the project UID/EEA/50008/2013.

\section{References}

1. Cimini, L, Jr. (1985). Analysis and simulation of a digital mobile channel using orthogonal frequency division multiplexing. IEEE Transactions on Communications, 33(7), 665-675.

2. 3rd generation partnership project: technical specification group radio access network; physical layers aspects for evolved UTRA (2006). 3GPPP TR 25.814.

3. IEEE Std 802.11a-1999 (Supplement to IEEE std.802.11-1999), The Institute of Electrical and Electronic Engineering, NY.

4. Digital Video Broadcasting (DVB); framing structure, channel coding and modulation for digital terrestrial television (2009), ETSI Standard: EN 300744 V1.6.1

5. O'Neill, R., \& Lopes. L. (1995). Envelope variations and spectral splatter in clipped multicarrier signals. In IEEE PIMRC'95, pp. 1-5.

6. Li, X., \& Cimini, L, Jr. (1998). Effects of clipping and filtering on the performance of OFDM. IEEE Communication Letters, 2(5), 131-133.

7. Müller, S., Bräuml, R., Fischer, R., \& Huber, J. (1997). OFDM with reduced peak-to-average power ratio by multiple signal representation. Annales of Telecommunications, 52(1), 58-67.

8. Cimini, L, Jr., \& Sollenberger, N. (1999). Peak-to-average power reduction of an OFDM signal using partial transmit sequences. IEEE Communication Letters, 4(3), 86-88.

9. Rahmatallah, Y., \& Mohan, S. (2013). Peak-to-average power ratio reduction in OFDM systems: A survey and taxonomy. IEEE Communication Surveys and Tutorials, 15(4), 1567-1592.

10. Han, S. J., \& Lee, J. H. (2005). An overview of peak-to-average power ratio reduction techniques for multicarrier transmission. IEEE Wireless Communications, 12(2), 56-65.

11. Raab, H., et al. (2005). Power amplifiers and transmitters for RF and microwave. IEEE Transactions on Microwave Theory Technology, 50(3), 814-826.

12. Dinis, R., \& Gusmão, A. (1996). CEPB-OFDM: A new technique for multicarrier transmission with saturated power amplifiers. In IEEE ICCS'96, pp. 1-5.

13. Thompson, S. C. (2005). Constant OFDM envelope phase modulation. Ph.D. Dissertation, University. California, San Diego, USA.

14. Thompson, S. C., et al. (2008). Constant envelope OFDM. IEEE Transactions on Communications, 56(8), 1300-1312.

15. Guerreiro, J., Dinis, R., \& Montezuma, P. (2015). Optimum performance and spectral characterization of CE-OFDM signals. In VTC Fall'15, pp. 1-5.

16. Guerreiro, J., Dinis, R., \& Montezuma, P. (2016). On the detection of CE-OFDM signals. IEEE Communication Letters, 20(11), 2165-2168.

17. Ayanoglu, E., Heydari, P., \& Capolino, F. (2014). Millimeter-wave massive MIMO: The next wireless revolution? IEEE Communications Magazine, 52(9), 56-62.

18. Peng, F., \& Ryan, E. (2014). MLSD bounds and receiver designs for clipped OFDM channels. IEEE Transactions on Wireless Communications, 7(9), 3568-3578.

19. Guerreiro, J., Dinis, R., \& Montezuma, P. (2013). Optimum and sub-optimum receivers for OFDM signals with strong nonlinear distortion effects. IEEE Transactions on Communications, 61(9), 3830-3840.

20. Guerreiro, J., Dinis, R., \& Montezuma, P. (2015). On the optimum multicarrier performance with memoryless nonlinearities. IEEE Transactions on Communications, 63(2), 498-509.

21. Tellambura, C. (2001). Computation of the continuous-time PAR of an OFDM signal with BPSK subcarriers. IEEE Communication Letters, 5(5), 185-187.

22. Bussgang, J. (1952). Crosscorrelation functions of amplitude-distorted gaussian signals. M.I.T. RLE Technical Report, no., 216, pp. 1-14. 
23. Rowe, H. (1982). Memoryless nonlinearities with gaussian input: Elementary results. Bell System Technical Journal, 61(67), 1519-1526.

24. Dardari, D., Tralli, V., \& Vaccari, A. (2000). A theoretical characterization of nonlinear distortion effects in OFDM systems. IEEE Transactions on Communications, 48(10), 1755-1764.

25. Banelli, P., \& Cacopardi, S. (2000). Theoretical analysis and performance of OFDM signals in nonlinear AWGN channels. IEEE Transactions on Communications, 48(3), 430-441.

26. Dinis, R., \& Gusmão, A. (2004). A class of nonlinear signal processing schemes for bandwidth-efficient OFDM transmission with low envelope fluctuation. IEEE Transactions on Communications, 52(11), 2009-2018.

27. Szego, G. (1975). The art of computer programming volume 23: Orthogonal polynomials. Providence: Colloquium Publications.

28. Dinis, R., \& Gusmão, A. (2004). Iterative receiver techniques for cancellation of deliberate nonlinear distortion in OFDM-type transmission. In IEEE International OFDM Workshop'04, pp. 1-5.

29. Tellado, J., Hoo, L., \& Cioffi, J. (2003). Maximum likelihood detection of nonlinearly distorted multicarrier symbols by iterative decoding. IEEE Transactions on Communications, 51(2), 218-228.

30. Abramowitz, M., \& Stegun, I. A. (1965). Handbook of mathematical functions. New York: Dover.
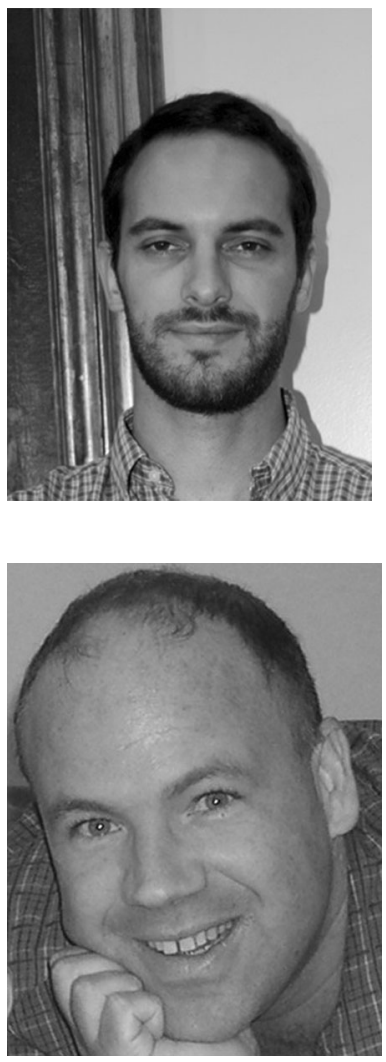

João Guerreiro received the M.Sc. Degree and the Ph.D. degree from Faculdade de Ciências e Tecnologia (FCT), Universidade Nova de Lisboa (UNL), in 2012 and 2016, respectively. He is currently a postdoc researcher at Instituto de Telecommunicações and assistant professor at Universidade Autónoma de Lisboa. His research interests include wireless communications and digital signal processing.

Rui Dinis received the Ph.D. degree from Instituto Superior Técnico (IST), Technical University of Lisbon, Portugal, in 2001 and the Habilitation in Telecommunications from Faculdade de Ciências e Tecnologia (FCT), Universidade Nova de Lisboa (UNL), in 2010. From 2001 to 2008 he was a Professor at IST. Currently he is an associated professor at FCT-UNL. During 2003 he was an invited professor at Carleton University, Ottawa, Canada. He was a researcher at CAPS (Centro de Análise e Processamento de Sinal), IST, from 1992 to 2005 and a researcher at ISR (Instituto de Sistemas e Robótica) from 2005 to 2008. Since 2009 he is a researcher at IT (Instituto de Telecomunicações). Rui Dinis is editor at IEEE Transactions on Communications (Transmission Systems-Frequency-Domain Processing and Equalization) and guest editor for Elsevier Physical Communication (Special Issue on Broadband Single Carrier Transmission Techniques). He has been actively involved in several international and national research projects in the broadband wireless communications area. His research interests include modulation, equalization, channel estimation and synchronization 


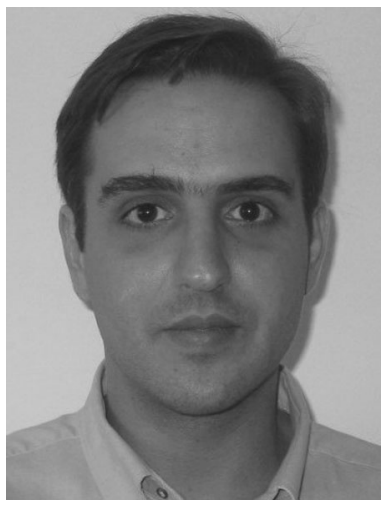

Paulo Montezuma received the Ph.D. degree from FCT-UNL (Faculdade de Ciências e Tecnologia da Universidade Nova de Lisboa), in 2007. Since 2001 he is teaching at the Department of Electrical Engineering (DEE) of the FCT. From 1996 to 2005 he was a researcher at CAPS/IST (Centro de Análise e Processamento de Sinais/Instituto Superior Técnico). In 2001 he joined the Uninova CTS research centre. $\mathrm{He}$ has been involved in several research projects in Telecommunications area, both national and European. His main research interests include modulation, channel coding, signal design for wireless communications, nonlinear effects and spread spectrum systems. 